\section{Desarrollo de una matriz conceptual para la innovación curricular en profesiones de la salud}

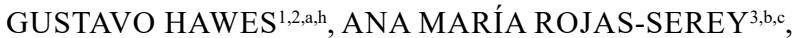 \\ MÓNICA ESPINOZA ${ }^{2,4, c, d}$, SANDRA OYARZO ${ }^{2,4, c, d}$, \\ SILVANA CASTILLO-PARRA ${ }^{5, \mathrm{e}, \mathrm{f}}$, MANUEL CASTILLO ${ }^{2, \mathrm{a}, \mathrm{f}}$, \\ LUIS ROMERO ${ }^{2,6, \mathrm{~g}}$
}

\section{Concept frame for curricular innovation in health care schools}

We herein describe the conceptual dimension of the curricular innovation process carried out in the Faculty of Medicine of the University of Chile. We describe the context of innovation. The theoretical pertinence and relevance of a competence driven curriculum for health care professionals is discussed. The epistemological, ontological and didactic dimensions of the curricular innovation are examined. A main issue is the notion of competence and its significance in professional training. The curriculum is essentially considered as a moral endeavor, especially for health care professionals and their quest to improve the quality of life of the population.

(Rev Med Chile 2017; 145: 1193-1197)

Key words: Curriculum; Chile; Education, Medical; Health Occupations.

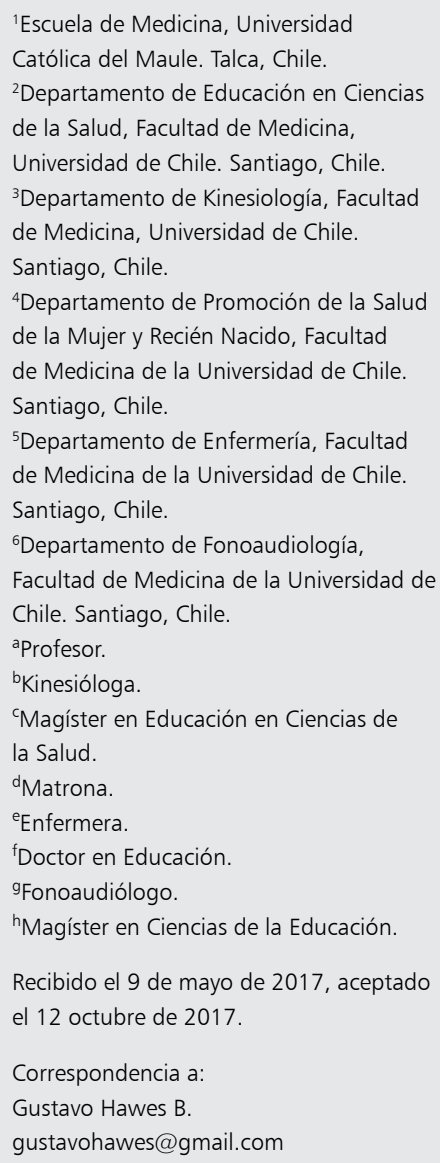

L os procesos de innovación curricular en la educación superior chilena han utilizado una matriz conceptual con lógicas de la iniciativa Alpha-Tuning ${ }^{1,2}$. En el caso de la Facultad de Medicina de la Universidad de Chile se configuran y articulan diversos elementos que constituyen una matriz conceptual propia ${ }^{3}$.

El proceso de innovación desarrollado, es eminentemente moral, más que sólo científico o tecnológico, tanto por la labor universitaria de recuperación de la democracia, como por una reacción ante la concepción reduccionista del modelo de competencias promovido desde el EEES5. Para la formación médica, los "valores profesionales, personales y educacionales [son] la fuerza impulsora más significativa para todos los implicados, sean o no conscientes de ello"(6:6).

\section{Antecedentes contextuales}

La Universidad de Chile es una institución pública y compleja, con aproximadamente 39.000 estudiantes. Desde 2006 se compromete con el modelo de formación basada en competencias a través del Consejo de Rectores de las Universidades Chilenas, iniciando un proceso de innovación curricular, formalizado en el Plan de Desarrollo Institucional (PDI). En el caso de la Facultad de Medicina, diversos factores se combinan en la génesis del mismo: bases de política nacional e institucional según permite distinguir su Modelo Educativo, y cuyas orientaciones están contenidas en su PDI.

En la búsqueda de una matriz conceptual y metodológica, en 2007 la Dirección de Pregrado de la Vicerrectoría de Asuntos Académicos, y la 
Facultad de Medicina iniciaron procesos de innovación y cambios curriculares bajo el paradigma del currículum orientado a competencias, lo cual significó un giro radical respecto de la manera tradicional de construir el currículo.

La innovación curricular se basa en iniciativas previas de la Facultad, políticas institucionales del PDI, propuestas en documentos de trabajo de Vicerrectoría de Asuntos Académicos, así como otros proyectos financiados por el Ministerio de Educación. Finalmente, la discusión y reflexión de la comunidad universitaria en torno a la innovación es el espacio que mayor riqueza proporciona a la matriz conceptual, tanto por la variedad de perspectivas como por la dinámica misma de construcción del conocimiento.

\section{Argumentación teórica para un currículum basado en competencias}

Teoría es concebida como una construcción social y colectiva ${ }^{6}$ antes que un corpus externo, universal e impositivo. Los conceptos teóricos iniciales vienen dados por las referencias al paradigma de la complejidad ${ }^{7}$ así como otras propuestas en torno al tema de las competencias.

Una crítica a las currícula es su configuración a partir de unidades de conocimiento relativamente independientes ${ }^{8}$, cuya articulación está dada por la concepción del conocimiento profesional como una combinación de saberes que, luego, el profesional deberá integrar en soluciones específicas. La fragmentación del saber, resultante de la hiperespecialización, explica la débil conexión interna del currículum de formación profesional.

Morin califica de patológica la situación contemporánea del conocimiento, por la excesiva simplificación que hace invisible la real complejidad, señalando que la dificultad del pensamiento complejo es que debe afrontar las interacciones infinitas, la incertidumbre y la contradicción ${ }^{7}$.

Desde la mirada de las profesiones y la formación, la competencia se concibe como "saber actuar", noción clave en el modelo de la Facultad, definiendo competencia como "saber actuar de manera pertinente en diversas situaciones y contextos, para enfrentar problemas propios de la profesión y la ciudadanía con claros criterios de calidad y humanidad, para lo cual se seleccionan y movilizan recursos internos (conocimientos, experiencias, etc.), de contexto y de redes (de datos, de personas), estando en condiciones de dar razón de las decisiones y actuaciones, y haciéndose cargo de los efectos e impactos de las mismas" (Modelo Educativo de la Facultad de Medicina). Esta noción integra propuestas de diversos autores, pero principalmente se apoya sobre las orientaciones de la universidad respecto de atender a las necesidades del proyecto país, siguiendo la senda trazada por el discurso inaugural de Andrés Bello ${ }^{11}$.

\section{Características de la gestión para la construcción curricular}

\section{Transparencia}

Los procesos de construcción son abiertos parcialmente a las comunidades involucradas, dependiendo del estado de avance de las propuestas, habiendo grados de transparencia: en el marco de las comisiones de trabajo, del claustro, de la sociedad.

\section{Participación}

La construcción curricular demanda participación de los principales actores del proceso formativo: los responsables político-administrativos de la institución, los académicos, los estudiantes, la sociedad representada por quienes se verán afectados de una u otra forma por las propuestas formativas.

\section{Validez curricular}

Juicio que establece el grado de convergencia entre una propuesta formativa y los criterios para su evaluación, adoptando significaciones variadas: institucional, social, académica.

\section{Dimensiones de la innovación curricular}

La innovación curricular desarrollada en la Facultad de Medicina, contempla tres dimensiones que son necesarias, ya que en su conjunto brindan sustento y coherencia a dicho proceso, resguardando que los cambios no se reduzcan a aspectos técnicos o precedimentales. Estas nociones y significados son descritos en la Tabla 1. A saber, Dimensión Epistemológica, Ontológica y Didáctica. 
Tabla 1. Dimensiones de la Innovación Curricular

\begin{tabular}{|lll|}
\hline Dimensión & Noción & Significado curricular \\
\hline Epistemológica & $\begin{array}{l}\text { Concepción sobre el } \\
\text { conocimiento válido } \\
\text { al interior de una } \\
\text { comunidad profesional o } \\
\text { académica }\end{array}$ & $\begin{array}{l}\text { La disposición de espacios locales para articular diálogos en conversación } \\
\text { con otras propuestas, visiones y posiciones, incluyendo las teorías generales, } \\
\text { permiten las construcciones locales y contextualizadas del conocimiento, } \\
\text { que no son consideradas como universales, pero pueden ser transferible a } \\
\text { otras realidades }\end{array}$ \\
\hline Ontológica & $\begin{array}{l}\text { Concepción del ser y su } \\
\text { construcción }\end{array}$ & $\begin{array}{l}\text { La universidad construye un discurso que configura realidades, sociales, } \\
\text { tecnológicas, y también personales donde se conjugan pluralismo, libertad } \\
\text { y multidimensionaidad, entendiendo formación como forma de ser en la } \\
\text { ciudadanía }\end{array}$ \\
\hline Didáctica & $\begin{array}{l}\text { Concepción de la relación } \\
\text { docente-estudiante en el } \\
\text { proceso formativo }\end{array}$ & $\begin{array}{l}\text { Centrada en el que aprende, promoviendo el compromiso con su propio } \\
\text { desarrollo. Rol docente: facilitar el aprendizaje, reconociendo que esto } \\
\text { ocurre en profundidad cuando hay una construcción activa de sentido en el } \\
\text { estudiante como protagonista de este proceso }\end{array}$ \\
\hline
\end{tabular}

\section{Discusión}

Las orientaciones provenientes del programa Tuning en el EEES se asocian principalmente a las necesidades de la industria. Se entiende así que la formación debe alinearse con las demandas actuales y emergentes de la industria en sus diversas expresiones ${ }^{2}$. Frente a esta tendencia, las críticas se centran en el carácter reductivo y funcionalista que ellas implican para la educación superior, especialmente lo que Barnett ${ }^{10}$ califica como "universidad empresa", señalando que considera que lo suyo tiene que ver con servicios y productos de formación y transmisión del conocimiento con valor de cambio en el mercado (p. 6). En esta tensión, la propuesta de la innovación curricular en la Facultad de Medicina se inclina por una noción que considera que el referente de la formación no son los requerimientos de la industria, sino que las necesidades del país y la sociedad, sintetizados en el término "civismo". Esto requiere una visión más compleja que simple, más reflexiva que técnica, más política que funcional.

Atendiendo a lo anterior, la construcción del currículum supone un tipo de conocimiento, el cual es concebido como un objeto en movimiento, dinámico y no lineal, en interacción permanente con sus diversas fuentes y actores, en lo que Green ${ }^{14}$ denomina un currículum hipervinculado. Además, su construcción demanda un modelo participativo y transparente que garantice no sólo la validez sino también la legitimidad de la propuesta formativa.
Un tercer elemento que surge al confrontar la propuesta de la Facultad de Medicina con otros modelos de formación profesional, se relaciona con la aceptación de la divergencia como un principio que colisiona y a la vez convive con la convergencia ${ }^{15}$. Si bien es cierto que deben asegurarse a la sociedad y a la fe pública los niveles de logro y competencia que den garantías acerca de la formación, también es claro que la autonomía, la creatividad y la versatilidad deben caracterizar a estos profesionales para hacerse cargo de los diversos contextos en que les toca actuar'.

Las cambiantes relaciones entre el Estado, las profesiones, los sujetos, y los diversos actores sociales $^{16}$ relevan la necesidad del cambio cultural, enfrentando los desafíos que provienen de las resistencias al mismo. En este marco, la innovación curricular será siempre un proceso político, social y moral.

La evidencia disponible en la literatura sobre el currículum de medicina indica que los cambios son una necesidad para la supervivencia de la profesión. Estos cambios se focalizan en competencias clave para mejoramiento de la calidad de la atención de salud prestadas a individuos, comunidades, sociedad nacional, considerando que las condiciones de vida de la población han cambiado de manera radical.

Hoy se demanda al currículum de las profesiones de la salud transitar hacia un modelo integrado, con un temprano acercamiento a la profesión como praxis, teniendo como norte las actuaciones profesionales, porque una auténtica 
formación sólo es posible en el marco del ejercicio, enfrentando con otros profesionales y actores la complejidad y relativa ambigüedad de las situaciones reales.

\section{Conclusiones}

La experiencia de la Facultad de Medicina de la Universidad de Chile se traduce en ocho carreras rediseñadas y funcionando alineadas al modelo impulsado institucionalmente, lo que permite concebir que cualquier iniciativa de innovación curricular requiere una matriz conceptual que sirva de referencia para articular y dar sentido al conjunto de los diversos elementos que la componen. La ausencia de una matriz conceptual definida, discutida y negociada promueve los procedimientos técnicos, asumiendo que el dispositivo técnico es una manera de evitar o prescindir de la discusión conceptual.

Tratándose de una construcción de este tipo, es necesaria la conversación reflexiva para la construcción de la innovación curricular. Un primer punto a dilucidar es el de la noción de competencia que sustentará teóricamente el trabajo, determinando el sentido profundo del currículum. Adherir a una visión mecanicista y fragmentaria de la competencia no es la opción de la Facultad de Medicina de la Universidad de Chile. Su propuesta se orienta hacia una noción que en la práctica se convierte en un discurso político tanto como pedagógico. En lo político se hace una opción por entender la competencia en el marco de la formación de profesionales como una forma de ser en la ciudadanía. En lo pedagógico, se entiende la competencia como el saber actuar en contextos complejos y fluidos, movilizando recursos de diversa naturaleza y haciéndose responsable de los resultados e impactos de sus decisiones en la salud de las personas.

Hay garantías que deben darse precisamente para evitar la improvisación o que estos cambios sean considerados propios de unos cuantos iluminados. Tampoco puede ser un acto cosmético, o una disposición autoritaria.

Las garantías para la innovación tienen que ver con transparencia, participación y validez. Este diálogo es transparente en cuanto se hace visible y tienen acceso a la misma los miembros de la comunidad. Es participativa porque las decisiones son producto del acuerdo entre docentes, estudiantes y administradores. Su validez debe estar garantizada por la reflexión y la negociación de sentido entre los diversos participantes.

Desde la perspectiva del producto mismo"currículum y plan de formación" se necesita también garantizar su legitimidad ante la comunidad, es decir, las bases sobre las que se sustenta, los modos de producción de conocimiento y toma de decisiones, así como la capacidad de ser sometido a prueba.

Organizacionalmente, se esperan garantías relacionadas con el aseguramiento del tiempo protegido, el reconocimiento del trabajo, y la asignación de recursos. Todas estas tienden a proporcionar bases y respaldos para desarrollar los procesos de innovación curricular. También se incorporan las condiciones para la instalación cultural e implementación de las currícula. El liderazgo institucional debe asegurar su viabilidad no sólo en el discurso, sino que en el efectivo compromiso con los cambios requeridos.

\section{Referencias}

1. González J, Wagenaar R. Quality and European Program Design in Higher Education. European Journal of Education 2003; 38 (3): 241-51.

2. González J, Wagenaar R. (Eds.). Tuning Educational Structures in Europe. Informe Final. Fase Uno. Bilbao (España): Universidad de Deusto y Universidad de Groningen, 2003.

3. Schiro M. Curriculum Theory. Conflicting Visions and Enduring Concerns: Sage Publications. 2008.

4. Barnett WP. Organizational Change. Annual Review of Sociology, 1995; 21 (1): 217-236. En http:// www.annualreviews.org/doi/abs/10.1146/annurev. so.21.080195.001245.

5. Fish D, Coles C. Medical education. Developing a curriculum for practice. Berkshire, UK: Open University Press. 2005.

6. Gibbons, M. The University as an Instrument for the Development of Science and Basic Research: The Implications of Mode 2 Science. En D. D. Dill \& B. Sporn (Eds.) Emerging Patterns of Social Demand and University Reform: Through a Glass Darkly. London: Pergamon, International Association of Universities. 1995: 90-104.

7. Morin E. La necesidad de un pensamiento complejo. En S. González Moena (Ed.), Pensamiento complejo. En torno a Edgar Morin, América Latina y los procesos educativos. Santafé de Bogotá: Magisterio. 1991. 
8. Jonnaert P, Masciotra D, Barrette J, Morel D, Mane Y. From Competence in the Curriculum to Competence in Action. Prospects 2007; 37 (2): 187-203.

9. Le Boterf G. Construire les compétences individuelles et collectives. La compétence n'est plus ce qu'elle etait Paris: Editions des Organisations. 2004.

10. Barnett R, Parry G, Coate K. Conceptualising Curriculum Change Teaching in Higher Education 2001; 6 (4): 435-49.

11. Bello A. Discurso Inaugural de la Universidad de Chile. Pronunciado en la instalación de la Universidad de Chile el día 17 de septiembre de 1843. Santiago de Chile: Universidad de Chile. 1843.

12. Walkington, J. A process for curriculum change in engineering education European Journal of Engineering Education 2002; 27 (2): 133-48. En http://www.tandfonline.com/doi/abs/10.1080/03043790210129603
13. Barnett RW. Imagining the university. International Higher Education 2013; 71: 6-8.

14. Green KH. Participating in the Hyperlinked Curriculum. En L. Kattington (Ed.), Handbook of Curriculum Development. New York, NY: Nova Science Publishers, Inc. 2010: 135-175. En http://daneshnamehicsa.ir/ userfiles/file/Resources/Intro-HANDBOOK\%20OF\%20 CURRICULUM\%20Development.pdf

15. Singh H, Singh A. Principles of Complexity and Chaos Theory in Project Execution: A New Approach to Management. Cost Engineering, AACE 2002; 44 (12): 23-33.

16. Beach D. Some General Developments in the Restructuring of Education and Health Care Professions in Europe. En I. Goodson \& S. Lindblad (Eds.), Professional Knowledge and Educational Restructuring in Europe. Rotterdam, The Netherlands: Sense Publishers. 2011: 25-39. 